\title{
Awareness of Intraoral Scanners and Knowledge of Effects of Different Lights on the Accuracy of Intraoral Scanners Among Dental Students and Practitioners
}

\author{
Aman Merchant ${ }^{1}$, Deepak Nallaswamy ${ }^{2}$ and Subhabrata Maiti ${ }^{3}$ \\ ${ }^{1}$ Post Graduate Saveetha Dental College and Hospitals Saveetha Institute of \\ Medical and Technical Sciences Saveetha University, Chennai, India \\ ${ }^{2}$ Professor and Director of Academics, Department of Prosthodontics Saveetha Dental College and \\ Hospitals Saveetha Institute of Medical and Technical Sciences Saveetha University, Chennai, India \\ ${ }^{3}$ Assistant Professor Department of Prosthodontics Saveetha Dental College and Hospitals \\ Saveetha Institute of Medical and Technical Sciences Saveetha University, 162, Poonamallee \\ High Road Chennai-600077 Tamil Nadu, India
}

\section{ABSTRACT}

Digital dentistry is an emerging branch which aims on improving the efficiency of the outcome of the treatment. One of the newly used instruments which has gained a lot of popularity is an intraoral scanner. An intraoral scanner helps in recording digital impressions which overcomes the limitations of conventional impressions. Hence, it is important to increase the awareness about all the aspects of intraoral scanner to utilize it to its full potential. The objective of this study is to evaluate the knowledge and awareness of intraoral scanners and the effects of different lights on its accuracy among dentists. The present study was done among the dental practitioners and dental students in India. A questionnaire was made and given to the dentist to check their knowledge and awareness about intraoral scanners and the effects of different lights on its accuracy. It was found that most of the dental practitioners and dental students had knowledge about intraoral scanners but were not aware about the limitations and effects of different lights on the accuracy of intraoral scanners. The data obtained from the above study shows that the dentists are not aware of the effects of different lights on accuracy of different intraoral scanners and need to be educated on the same for obtaining better results.

\section{KEY WORDS: INTRAORAL SCANNERS, LIGHT, ACCURACY.}

\section{INTRODUCTION}

Digital dentistry is an emerging avenue in the field of dentistry. It refers to the use of digital technology and computer aided systems to carry out dental procedures in contrast to the manual methods (Neville and van der

\section{ARTICLE INFORMATION}

*Corresponding Author: amanmrchnt@gmail.com

Received 13th June 2020 Accepted after revision 8th August 2020

Print ISSN: 0974-6455 Online ISSN: 2321-4007 CODEN: BBRCBA

Thomson Reuters ISI Web of Science Clarivate Analytics USA and Crossref Indexed Journal

\section{Clarivate
Analytics}

\section{rrossef}

NAAS Journal Score 2020 (4.31) SJIF: 2020 (7.728)

A Society of Science and Nature Publication,

Bhopal India 2020. All rights reserved.

Online Contents Available at: http//www.bbrc.in/

Doi: http://dx.doi.org/10.21786/bbrc/13.7/16
Zande, 2020). It is gaining popularity because of its accuracy and efficiency when compared with mechanical tools. The digital revolution has made a significant impact in changing the workflow which in turn affects the operating procedures (Mangano, Shibli and Fortin, 2016). The need of the hour is digitizing our work to obtain maximum efficiency (Kudva, 2016). There are different tools which have helped make digital dentistry an easier podium to work with. One such equipment is the use of intraoral scanners. Intraoral scanners (IOS) are devices for recording direct optical impressions in dentistry (Mangano et al., 2017). Like other three dimensional scanners, intraoral scanners project a light source onto 
the object which has to be scanned (Zimmermann et al., 2015). It can be a dental arch, prepared tooth or scan bodies of implants (Martin et al., 2015). The images of the dentogingival system or the scan bodies are captured by the sensors and processed by the sensors in the form of point clouds. The cloud points are then analyzed by the software which makes it into 3D models (Imburgia et al., 2017).

Advantages of using optical impression with intraoral scanners

- Less discomfort for the patient(Means and Flenniken, 1970; Ahlholm et al., 2018)

- $\quad$ Time efficient(Yuzbasioglu et al., 2014; Burhardt et al., 2016)

- $\quad$ Better communication with the patient and dental technician(Kugel, 2014; Lawson and Burgess, 2015)

- $\quad$ No errors due to errors in cast pouring(Patzelt et al., 2014; Joda and Brägger, 2015)

- $\quad$ The procedure becomes simplified(Park et al., 2015; Lecocq, 2016)

Disadvantages of using optical impression with intraoral scanners

- Subgingival finish lines are difficult to detect(Mandelli et al., 2017; Lim et al., 2018)

- Costly and difficult to maintain(Aragón et al., 2016)

The main feature of an intraoral scanner is its accuracy (Goracci et al., 2016)(Chochlidakis et al., 2016). There are certain factors that affect the accuracy of the intraoral scanners (Patzelt et al., 2013; Rutkanas et al., 2017). Firstly, the saliva plays an important role in scanning with an intraoral scanner as it can wash away the spray particles and reduce the efficiency. Also, there can be some error in scanning as the saliva film created on the tooth will cause misreading of the object geometry (Kurz, Attin and Mehl, 2015). Secondly, the scanning protocol plays an influential role. Inadequate scanning leads to errors in the final prosthesis (Müller et al., 2016). Thirdly, the ambient light affects the coordinates measured by the intraoral scanner (Blanco et al., 2009). This survey was conducted to know about the awareness of intraoral scanners and the effects of different lights on the accuracy of intraoral scanners among dental practitioners and dental students.

\section{MATERIAL AND METHODS}

A questionnaire survey was conducted among different dental practitioners and dental students in India during January, 2020. The questionnaire was formulated in google forms. A total of 289 subjects were sent the questionnaire consisting of questions regarding the awareness about intraoral scanners and the effects of different lights on the accuracy of intraoral scanners out of which 117 subjects responded. Validation was done among post graduate students of the department of prosthodontics in XYZ Dental College. Ethical approval was obtained from the ethical board of XYZ Dental College. A simple random sampling was done. The survey was sent online to individuals and none were forced to fill the questionnaire. Guidelines followed as per the Helsinki declaration(Kemperman, 1982).

All the data was collected in Google sheets and analyzed using SPSS 21 (SPSS Inc., Chicago, IL). Chi square test was done to statistically analyze the data and visual representations of the data were plotted with the extracted data. The independent variables in this survey would be sex of the subject, the intraoral scanner used and the light used while using the scanner. The dependent variable in this survey would be the age and experience of the subject.

\section{RESULTS AND DISCUSSION}

As the application of digital models has broadened into many areas, the accuracy of 3D images acquired on an intraoral scanner has become an important topic in research (Auskalnis et al., 2019). Accuracy refers to precision and trueness of the object (Chandran et al., 2010). Hence it is important to adjust all the factors influencing the accuracy of intraoral scanners to maximize the effect. Ambient light has a great impact on the accuracy of the intraoral scanner (Revilla-León et al., 2020). Several in vitro studies have demonstrated the effect of ambient light on the accuracy of different intraoral scanners (Revilla-León et al., 2019a, 2019b). Based on these reports, a scanning accuracy difference of 37\%-44\% can be seen when under different light conditions(Revilla-León et al., 2019a).

A total of 289 subjects were sent the questionnaire out of which 117 subjects responded. Out of the 117 subjects, 70 (59.8\%) were females and 37 (40.2\%) were males. The mean age range of the study subjects were from 18 to 75 years. Out of the 117 responses, 35.5\% were post graduate other than prosthodontics, $31.6 \%$ were general practitioners, $22.8 \%$ were prosthodontics postgraduate, $8.5 \%$ students were undergraduate students and remaining were prosthodontist). Out of the 117 responses, 70.0\% were aware about the use of intraoral scanners, 20.0\% were not aware about the use of intraoral scanners and 10.0\% were aware about intraoral scanners to some extent (Figure 1). Out of 117 responses, 83 $(70.1 \%)$ were not aware about the effects of different lights on the accuracy of intraoral scanners, 25 (21.4\%) were aware about the effects of different lights on the accuracy of intraoral scanners to some extent whereas only $9(7.7 \%)$ were aware about the effects of different lights on the accuracy of intraoral scanners (Figure 10). There was a statistically significant difference in the responses of the questions with respect to the practitioner groups $(\mathrm{p}<0.05)$.

In the current survey, most of the dental students and dental practitioners were aware about intraoral scanners (Figure 4), its indications (Figure 5) and its limitations (Figure 6). Now, when digital dentistry is taking over, it 
is important for all dentists to be well versed with the latest equipment and techniques. More than half of the dentists, who were given the questionnaire do not use intraoral scanners in their clinical practice (Figure 7). With the use of intraoral scanners, the workload becomes quite dwindled. Hence, more number of clinicians should use intraoral scanners to reduce the workload and increase the efficiency. More than half of the dentists, who were given the questionnaire were not aware about the effects of different lights on the accuracy of intraoral scanners. Without proper knowledge, using any equipment can lead to errors and hence can negatively impact the results. Each scanner has its own advantages and disadvantages which a clinician should be aware of before using them. One of them is the effect of different lights on the accuracy of intraoral scanners. It is important to know about all the factors to maximize the potential of the system.

Table 1. Showing the correlation between different groups of dental practitioners and the awareness about intraoral scanners.

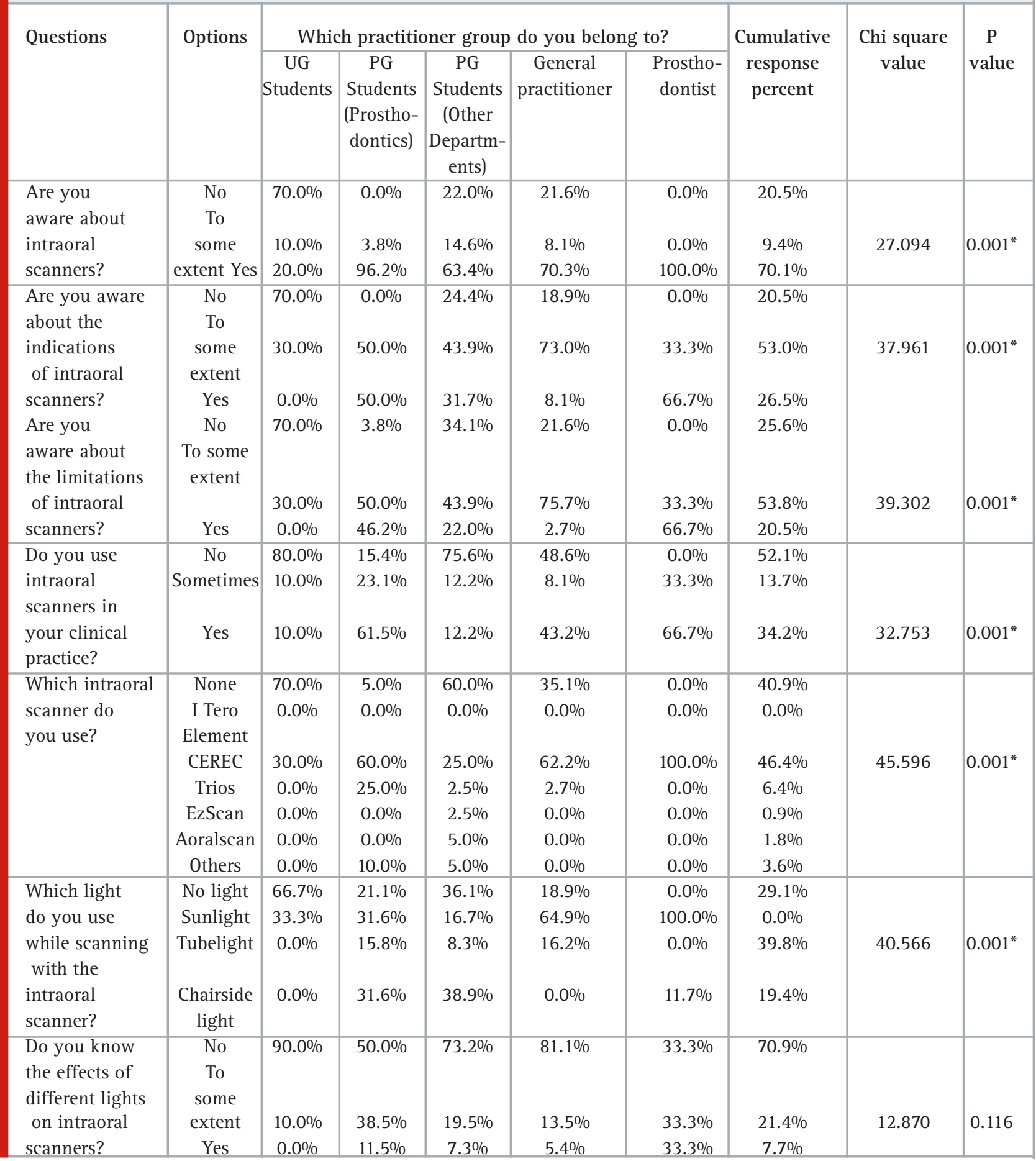




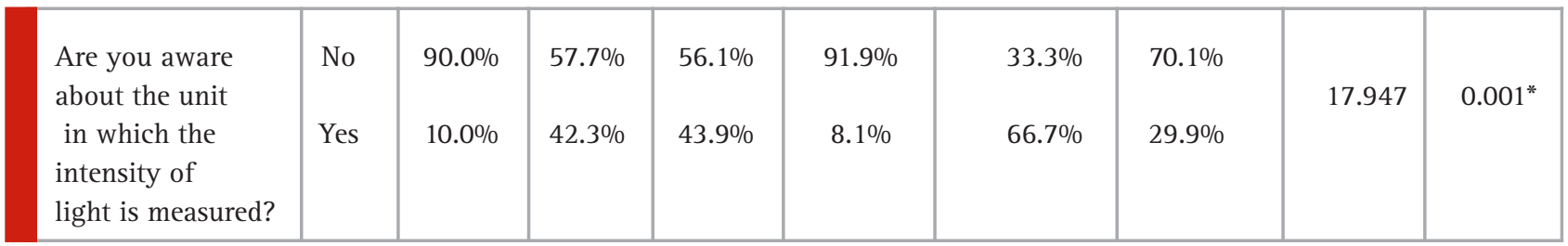

Figure 1: Bar graph representing the association between awareness about intraoral scanners and the practitioner group.

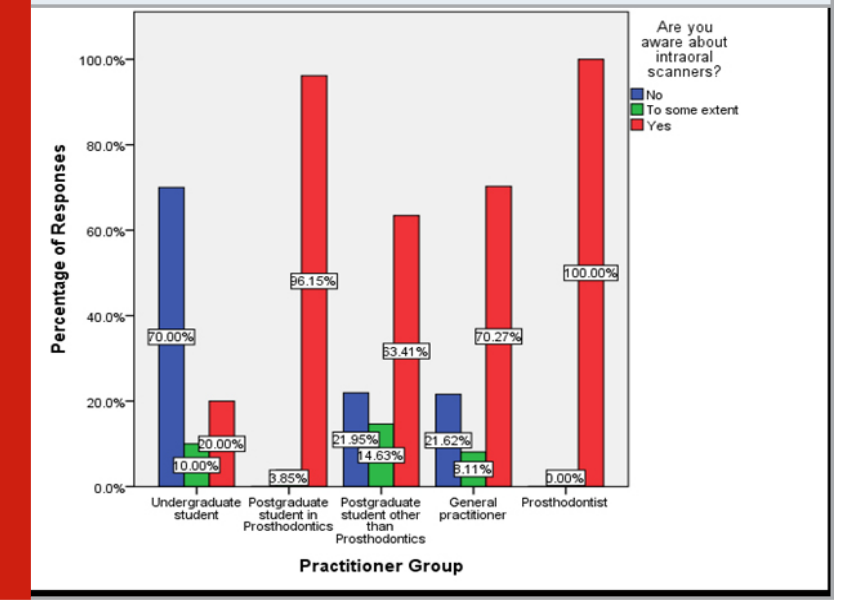

Figure 2: Bar graph representing association between usage of intraoral scanners in clinical practice and practitioner groups

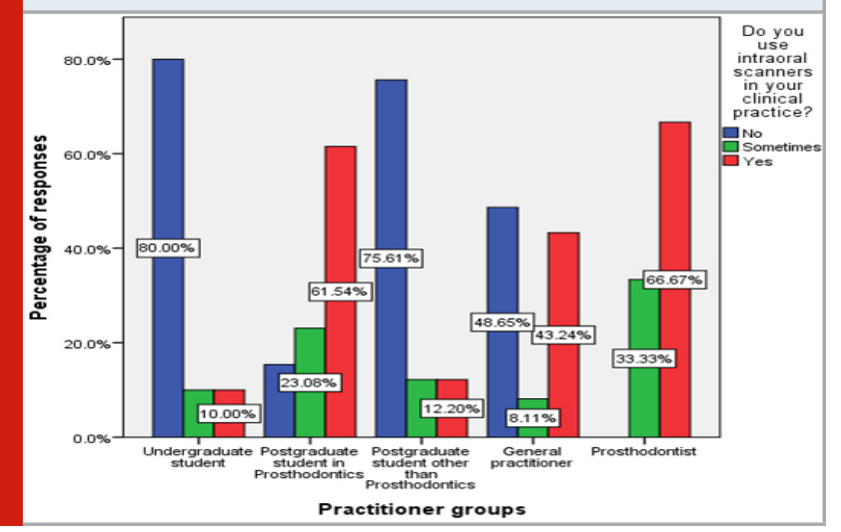

In one study, it showed that iTero Element, using chair and room light conditions resulted in better accuracy, whereas, for CEREC Omnicam, zero light resulted in better accuracy and for TRIOS 3, room light gave the best accuracy(Revilla-León et al., 2019a). In a similar study by Arakida et al(Arakida et al., 2018), they concluded that the most accurate results with a high-accuracy noncontact three-dimensional coordinate measuring machine like Infinite focus G5 was seen with 500 lux and 2500 lux of light. The 500 lux condition represents the illumination by a room light in a clinic, while the 2500 lux condition represents the illumination by a dental unit light(Viohl, 1979). Clinicians should understand the ambient lighting condition as a critical influencing factor on the scanning accuracy of IOSs, and a light meter should be included into the armamentarium of the digital device.

Although this study gives adequate information on the awareness regarding the intraoral scanners, there are limitations to the study. The major limitation is that the number of people who responded were very less. Additional studies are needed to fully understand the impact of lighting conditions on the accuracy of the available intraoral digitizer systems.

Figure 3: Bar graph representing the association between awareness of effects of different lights on intraoral scanners and practitioner groups.

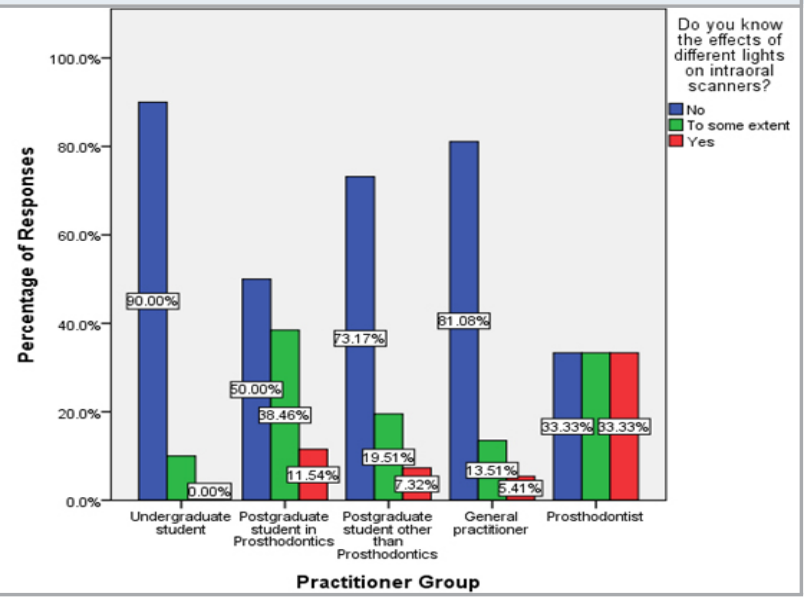

\section{CONCLUSION}

The data obtained from the above study shows that the dentists, mostly the undergraduate students and postgraduate students other than prosthodontics are not aware of the effects of different lights on accuracy of different intraoral scanners. It is very important for dental practitioners and students to know this as it can affect the outcome of the final prosthesis. The dental students and practitioners should be educated about the different aspects of intraoral scanners so that they can harness the full potential of intraoral scanners with minimum errors. The undergraduate and postgraduate students should be taught about the latest equipment and their proper use in their curriculum and there should be more lectures and seminars by the speakers for increasing the awareness about the proper use of latest techniques and equipment among the dental practitioners. More webinars and online lectures should be conducted for dental practitioners who are not able to attend the seminars. Critical analysis of the topic and panel discussion should be arranged for better understanding of the topic. After any lecture taken in 
a conference, a written questionnaire should be given to the audience to verify their understanding about the topic of the lecture. More frequent surveys should be circulated in the colleges to increase the awareness among the students.

\section{REFERENCES}

Ahlholm, P. et al. (2018) 'Digital Versus Conventional Impressions in Fixed Prosthodontics: A Review’, Journal of prosthodontics: official journal of the American College of Prosthodontists, 27(1), pp. 35-41.

Aragón, M. L. C. et al. (2016) 'Validity and reliability of intraoral scanners compared to conventional gypsum models measurements: a systematic review', European journal of orthodontics, 38(4), pp. 429-434.

Arakida, T. et al. (2018) 'Evaluating the influence of ambient light on scanning trueness, precision, and time of intra oral scanner', Journal of prosthodontic research, 62(3), pp. 324-329.

Auskalnis, L. et al. (2019) 'Effect of intraoral scanner, printer, and digital analog system on the accuracy of 3D printed models', Clinical Oral Implants Research, pp. 539-539. doi: 10.1111/clr.2_13510.

Blanco, D. et al. (2009) 'Influence Of Ambient Light On The Repeatability Of Laser Triangulation Digitized Point Clouds When Scanning EN AW 6082 Flat Faced Features'. doi: 10.1063/1.3273669.

Burhardt, L. et al. (2016) 'Treatment comfort, time perception, and preference for conventional and digital impression techniques: A comparative study in young patients', American journal of orthodontics and dentofacial orthopedics: official publication of the American Association of Orthodontists, its constituent societies, and the American Board of Orthodontics, 150(2), pp. 261-267.

Chandran, D. T. et al. (2010) 'Two- and three-dimensional accuracy of dental impression materials: effects of storage time and moisture contamination', Bio-medical materials and engineering, 20(5), pp. 243-249.

Chochlidakis, K. M. et al. (2016) 'Digital versus conventional impressions for fixed prosthodontics: A systematic review and meta-analysis', The Journal of prosthetic dentistry, 116(2), pp. 184-190.e12.

Goracci, C. et al. (2016) 'Accuracy, reliability, and efficiency of intraoral scanners for full-arch impressions: a systematic review of the clinical evidence', European journal of orthodontics, 38(4), pp. 422-428.

Imburgia, M. et al. (2017) 'Accuracy of four intraoral scanners in oral implantology: a comparative in vitro study', BMC oral health, 17(1), p. 92.

Joda, T. and Brägger, U. (2015) 'Digital vs. conventional implant prosthetic workflows: a cost/time analysis', Clinical oral implants research, 26(12), pp. 14301435.

Kemperman, C. J. F. (1982) 'HELSINKI DECLARATION', The Lancet, p. 220. doi: 10.1016/s0140-6736(82)91072-8. Kudva, P. (2016) 'Digital dentistry: The way ahead',
Journal of Indian Society of Periodontology, p. 482. doi: 10.4103/jisp.jisp_355_16.

Kugel, G. (2014) 'Impression-taking: conventional methods remain steadfast as digital technology progresses', The Compendium of continuing education in dentistry, 35(3), pp. 202-203.

Kurz, M., Attin, T. and Mehl, A. (2015) 'Influence of material surface on the scanning error of a powderfree 3D measuring system', Clinical oral investigations, 19(8), pp. 2035-2043.

Lawson, N. C. and Burgess, J. O. (2015) 'Clinicians reaping benefits of new concepts in impressioning', The Compendium of continuing education in dentistry, 36(2), pp. 152-153.

Lecocq, G. (2016) 'Digital impression-taking: Fundamentals and benefits in orthodontics', International orthodontics / College europeen d'orthodontie, 14(2), pp. 184-194.

Lim, J.-H. et al. (2018) 'Comparison of digital intraoral scanner reproducibility and image trueness considering repetitive experience', The Journal of prosthetic dentistry, 119(2), pp. 225-232.

Mandelli, F. et al. (2017) 'Improvement of a Digital Impression with Conventional Materials: Overcoming Intraoral Scanner Limitations', The International journal of prosthodontics, 30(4), pp. 373-376.

Mangano, F. et al. (2017) 'Intraoral scanners in dentistry: a review of the current literature', BMC oral health, 17(1), p. 149.

Mangano, F., Shibli, J. A. and Fortin, T. (2016) 'Digital Dentistry: New Materials and Techniques', International Journal of Dentistry, pp. 1-2. doi: $10.1155 / 2016 / 5261247$.

Martin, C. B. et al. (2015) 'Orthodontic scanners: what's available?', Journal of Orthodontics, pp. 136-143. doi: 10.1179/1465313315y.0000000001.

Means, C. R. and Flenniken, I. E. (1970) 'Gagging-a problem in prosthetic dentistry', The Journal of Prosthetic Dentistry, pp. 614-620. doi: 10.1016/00223913(70)90224-6.

Müller, P. et al. (2016) 'Impact of digital intraoral scan strategies on the impression accuracy using the TRIOS Pod scanner', Quintessence international , 47(4), pp. 343-349.

Neville, P. and van der Zande, M. M. (2020) 'Dentistry, e-health and digitalisation: A critical narrative review of the dental literature on digital technologies with insights from health and technology studies', Community dental health, 37(1), pp. 51-58.

Park, H.-R. et al. (2015) 'Changes in views on digital intraoral scanners among dental hygienists after training in digital impression taking, BMC oral health, 15(1), p. 151.

Patzelt, S. B. M. et al. (2013) 'Assessing the feasibility and accuracy of digitizing edentulous jaws', Journal of the American Dental Association , 144(8), pp. 914-920.

Patzelt, S. B. M. et al. (2014) 'The time efficiency of 
intraoral scanners', The Journal of the American Dental Association, pp. 542-551. doi: 10.14219/jada.2014.23. Revilla-León, M. et al. (2019a) 'Intraoral digital scansPart 1: Influence of ambient scanning light conditions on the accuracy (trueness and precision) of different intraoral scanners', The Journal of prosthetic dentistry. doi: 10.1016/j.prosdent.2019.06.003.

Revilla-León, M. et al. (2019b) 'Intraoral digital scans: Part 2-influence of ambient scanning light conditions on the mesh quality of different intraoral scanners', The Journal of prosthetic dentistry. doi: 10.1016/j. prosdent.2019.06.004.

Revilla-León, M. et al. (2020) 'Clinical Study of the Influence of Ambient Light Scanning Conditions on the Accuracy (Trueness and Precision) of an Intraoral Scanner', Journal of Prosthodontics, pp. 107-113. doi: 10.1111/jopr.13135.

Rutkunas, V. et al. (2017) 'Accuracy of digital implant impressions with intraoral scanners. A systematic review', European journal of oral implantology, 10 Suppl 1, pp. 101-120.

Viohl, J. (1979) 'Dental operating lights and illumination of the dental surgery', International dental journal, 29(2), pp. 148-163.

Yuzbasioglu, E. et al. (2014) 'Comparison of digital and conventional impression techniques: evaluation of patients' perception, treatment comfort, effectiveness and clinical outcomes', BMC oral health, 14, p. 10.

Zimmermann, M. et al. (2015) 'Intraoral scanning systems - a current overview', International journal of computerized dentistry, 18(2), pp. 101-129.

\section{APPENDIX \\ Questions asked}

1. Name

2. Sex

3. Age

4. Which of the practitioner groups you belong to?

5. Are you aware about intraoral scanners?

6. Are you aware about the indications of intraoral scanners?

7. Are you aware about the limitations of intraoral scanners?

8. Do you use intraoral scanners in your clinical practice?

9. Which intraoral scanner do you use?

10. Which light do you use while scanning with the intraoral scanner?

11. Do you know the effects of different lights on intraoral scanners?

12. Are you aware about the unit in which the intensity of light is measured? 